\title{
Alternative digital filtering scheme for LumiCal read-out
}

\author{
Evgenii Lutsenko ${ }^{1,2, *}$ \\ ${ }^{1}$ Department of General and Applied Physics, Moscow Institute of Physics and Technology \\ ${ }^{2}$ Dzelepov Laboratory of Nuclear Problems, Joint institute of Nuclear Research
}

\begin{abstract}
The LumiCal electromagnetic calorimeter is designed for the forward region of future electron-positron colliders, such as CLIC and ILC. It is intended to improve hermeticity of detectors by detecting electrons, positrons, and photons at small angles. Currently, the detector prototypes are studied in the beam test conditions. An important part of the signal processing is the digital filtering implemented in the read-out electronics, influencing the precision and quality of gathered data. In this article two schemes of digital filtering of gathered signal from test beam for the LumiCal detector prototype are presented.
\end{abstract}

\section{Introduction}

Future electron-positron colliders in the TeV range (ILC [1], CLIC [2]) are designed to provide access to possible physics effects beyond Standard Model or to confirm its reliability. The main tasks of $e^{+} e^{-}$colliders are: studies of the Higgs boson (in processes $e^{+} e^{-} \rightarrow f \bar{f} H$ ), high-precision measurements of the top quark properties, Supersymmetry studies. Electrons on small angles are necessary detected for research those processes. One of detectors is the electromagnetic calorimeter LumiCal [3], which prototype is currently being designed and tested.

\section{Test beam 2016 for LumiCal detector prototype}

LumiCal is an electromagnetic Si-W sandwich calorimeter at small angles $\left(\theta_{\text {inner }}=31 \mathrm{mrad}\right.$; $\theta_{\text {outer }}=77 \mathrm{mrad}-$ for ILD detector) [3]. LumiCal is designed for integrated luminosity measurement in linear collider experiments. The calorimeter design with a tile is shown in figure 1a. The prototype for test beam is served by two read-out APV25 chips [4] in order to be able to read out all pads of a sensor. One chip includes a preamplifier and shaper, an analog pipeline and a deconvolution filter for each of its 128 channels.

A schematic layout of the experimental setup for testing the prototype of the electromagnetic calorimeter LumiCal consisting of several plates which are located one after another is shown in figure $1 \mathrm{~b}$. The first two layers are "tracker" - a part of detector prototype, which has no tungsten. The LumiCal detector prototype itself is represented by six plates after the tracker. In total, 2048 channels for data reading were used in this experimental setup. An electron beam of $1-6 \mathrm{GeV}$ passes through the target and radiate bremsstrahlung photons. In a magnetic field (dipole magnet $1-13 \mathrm{kGs}$ ), these beams are separated and fall into the LumiCal detector prototype.

\footnotetext{
*e-mail: lutsenko.evg.ol@gmail.com
} 


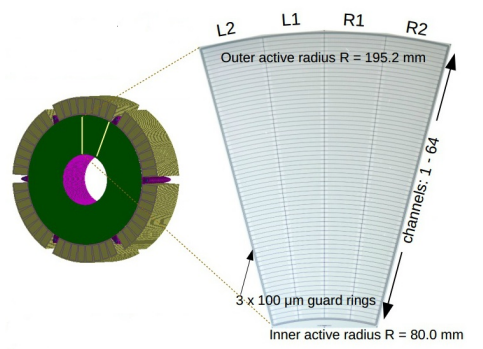

(a)

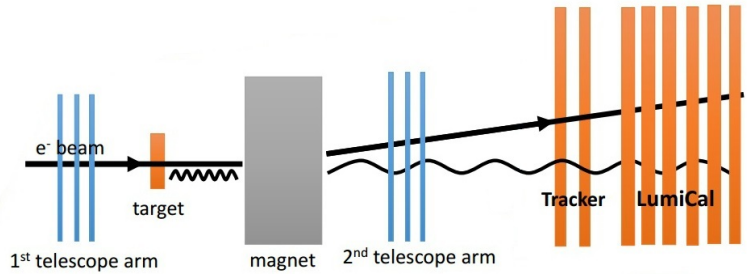

(b)

Figure 1: a) LumiCal design for ILD and one of its silicon-sensor tiles. b) A schematic layout (figure is not to scale) of the test beam 2016 for LumiCal.

\section{Digital filter schemes}

The readout scheme for time and amplitude measurements is presented in figure 2 . The scheme shows that sensor current is converted in analog signal after shaper, which produces a signal in the form of the various order $C R-R C^{n}$ filters, and after that it is digitized. After transformation, the signal has a similar amplitude as the charge value and signal-to-noise ratio is improved. Digital filtering is required to restore the original signal. Schematically this is shown on the right side of figure 2 .

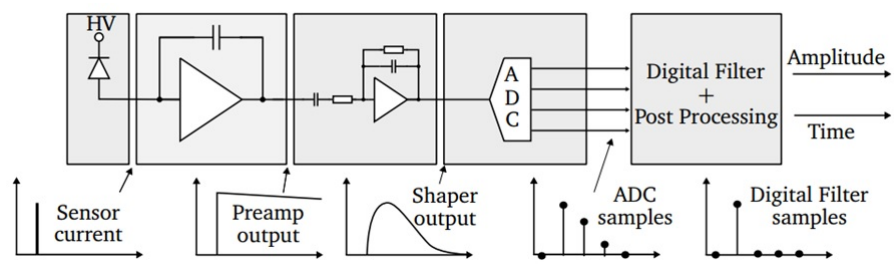

Figure 2: Block diagram of the proposed readout scheme [5].

\subsection{Fitting scheme}

During the beam test the readout electronics worked in asynchronous mode when the ADC clock was not synchronized with particles arrival. As a consequence, the signal is not sampled in its maximum.

For measuring the amplitude of the signal it was fitted by the $C R-R C$ filter response function which corresponds to the APV25 shaper implementation. The fit function (fig. 3a) is

$$
F(t)=A \frac{\left(t-t_{0}\right)}{\tau} e^{-\left(t-t_{0}\right) / \tau}
$$

where $\tau$ is shaping time, $t_{0}$ is pulse occurrence time, $A$ is the fit function amplitude and it is necessary to determine $A_{f i t}=A e^{-1}$. This Fitting scheme is a basic approach. 


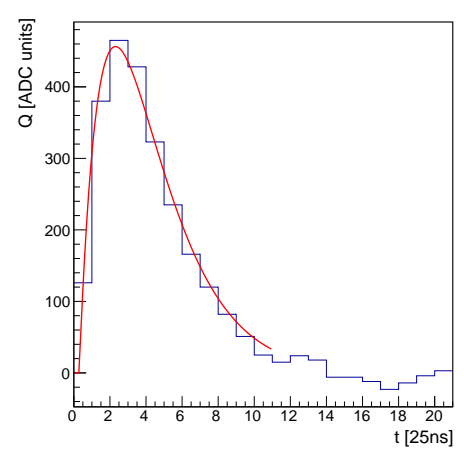

(a)

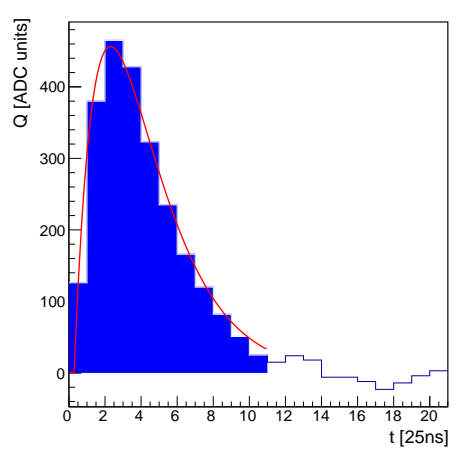

(b)

Figure 3: a) A signal of one channel with the result of fitting. b) The signal with the result of fitting (red line) and the area that is equal to the $\Sigma$ (filled area).

\subsection{Signal integration scheme}

An alternative to baseline approach of digital filtering was developed. It consists in parameterizing a maximum value of shaped signal $\left(Q_{\max }\right)$ of the waveform in each event with $\Sigma=\sum_{i=0}^{11} Q_{i}-$ the sum of the signal values in time for each event (fig. $3 \mathrm{~b}$ ). The sum of the signal values in time was taken for each event from 0 to 11 (out of 21) in order to reduce the effect of noise in a signal tail. For further analysis of the alternative digital filtering scheme, it is required to investigate only the peak of the signal (similar to section 3.1).

To compare the two quantities, the dependence of the maximum value $\left(Q_{\max }\right)$ on the sum of the signal values $(\Sigma)$ is plotted for all events of each channel. Each point in figure 4 corresponds to the signal from figure $3 \mathrm{~b}$ (max value of the signal versus the sum of its signal in time). The histogram must be constructed for each channel separately. After that, this dependence fits with the function $S(\Sigma)$.

$$
A_{\Sigma}=S(\Sigma)=B+\sqrt{C+D \Sigma}
$$

For fitting the dependencies presented in figure 4, many functions were tested, but the square root was chosen because it better than others describes the relationship between $Q_{\max }$ and $\Sigma$. The parameters $B, C, D$, in formula 2 , must be determined for each channel and run of the test beam separately. The red line in figure 4 corresponds to the fitting result of function (2) to the presented distribution of $Q_{\max }$ versus $\Sigma$. This function allows it to determine for each $\Sigma$ value its own signal amplitude $A_{\Sigma} . A_{\Sigma}$ is the amplitude of the signal for the developed alternative digital filtering scheme. This value is similar to $A_{f i t}$, but it is more averaged and less susceptible to various noises and errors since fitting (for determining $A_{\Sigma}$ ) is carried out for each channel, and not for each event in each channel (as in section 3.1 for $A_{f i t}$ ).

Similar to the Signal integration scheme, for the Fit scheme it is possible to calculate the integral of a function in a certain interval and compare it with the amplitude of the fit function $\left(A_{f i t}\right)$. The histogram will be almost identical to figure 4 .

The fitting (sect. 3.1) was taken as a standard to validate and compute efficiency of this digital filtering scheme. Because $A_{f i t}$ is increasing with the increase of the integral values, all range of $\Sigma$ was divided into some regions. Distribution of normalized residuals $(\epsilon)$ was 


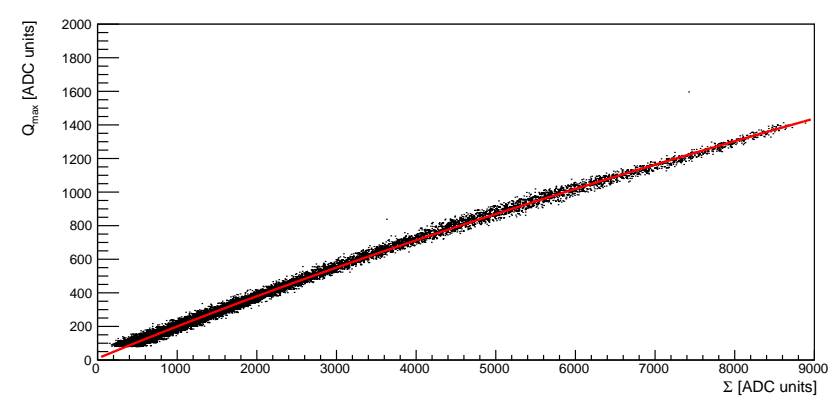

Figure 4: Correlation between the maximum value and the sum.

considered for them

$$
\epsilon=\frac{A_{\Sigma}-A_{f i t}}{A_{f i t}} .
$$

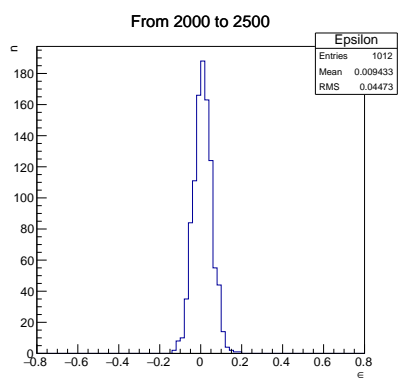

(a)

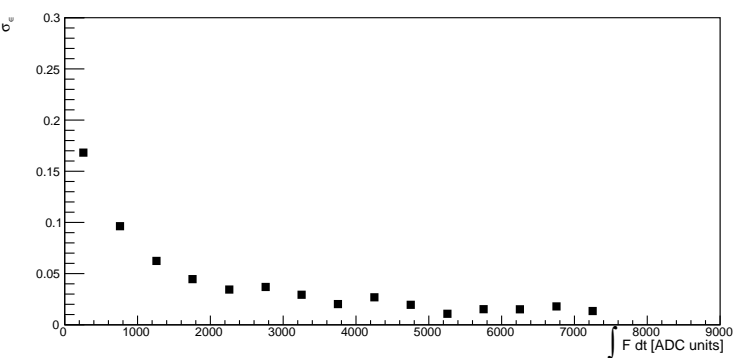

(b)

Figure 5: a) An example of distribution of normalized residuals (3) for one of the regions. b) Distribution of RMS for one channel as a function of $\Sigma$.

Example of normalized residuals distribution for one of the different regions for a channel is presented in figure 5a. Also, the resolution histograms were constructed for the signal integration digital filtering scheme. An example for one channel presented in figure $5 \mathrm{~b}$.

Correlation between $A_{f i t}$ and the difference of $A_{f i t}$ and $A_{\Sigma}$ is presented in figure 6. From the correlation it can be seen that the results are consistent with the baseline scheme and do not have systematic deviations.

As a result, the alternative method of digital filtering, which was developed, is well correlated with the fit method. Moreover, this method is about 150 times faster than the standard method. Alternative scheme of digital filtering (sect. 3.2) can be built into the detector electronics.

\section{Summary}

The test beam data of the electromagnetic calorimeter prototype LumiCal were processed by the basic (sect. 3.1) and alternative (sect. 3.2) methods of digital signal filtering. The 


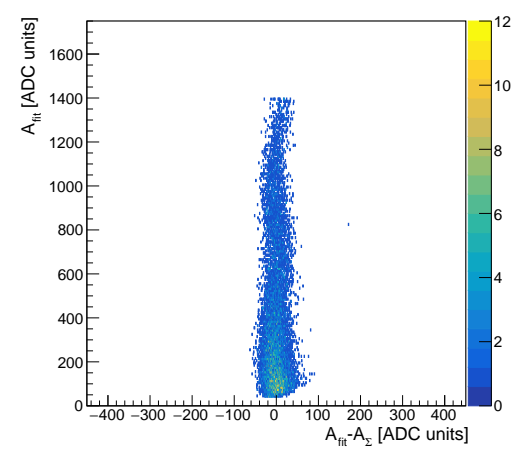

Figure 6: Correlation between $A_{f i t}-A_{\Sigma}$ and $A_{f i t}$.

developed Signal integration scheme proved to be quite effective. It correlates well with the fittings, processes the data 150 times faster and can be included in the electronics of the detector as a firmware.

\section{Acknowledgements}

The author is grateful to the supervision of A.Sapronov (JINR, Russia) and O.Borysov (Tel Aviv University, Israel) for execution of this work.

\section{References}

[1] Abramowicz, H. et al., Forward Instrumentation for ILC Detectors, JINST 5, P12002 (2010) doi:10.1088/1748-0221/5/12/P12002 [arXiv:1009.2433 [physics.ins-det]], 30 pages.

[2] Boland, M J et al.(CLIC and CLICdp Collaborations), Updated baseline for a staged Compact Linear Collider, (arXiv:1608.07537 [physics.acc-ph] CERN-2016-004), 57 pages.

[3] Abramowicz et al. [FCAL Collaboration], Measurement of shower development and its Molière radius with a four-plane LumiCal test set-up, Eur. Phys. J. C 78 (2018) no.2, 135 doi:10.1140/epjc/s10052-018-5611-9 [arXiv:1705.03885 [physics.ins-det]], 18 pages.

[4] French et al., Design and results from the APV25, a deep sub-micron CMOS front-end chip for the CMS tracker, Nucl. Instrum. Meth. A 466, 359 (2001). doi:10.1016/S01689002(01)00589-7, 7 pages.

[5] Szymon Kulis, Development of prototype luminosity detector modules for future experiments on linear colliders (Doctoral dissertation, Cracow, 2012), 175 pages. 